\title{
A THREE-POINT BOUNDARY VALUE PROBLEM WITH AN INTEGRAL CONDITION FOR A THIRD-ORDER PARTIAL DIFFERENTIAL EQUATION
}

C. LATROUS AND A. MEMOU

Received 9 February 2004

We prove the existence and uniqueness of a strong solution for a linear third-order equation with integral boundary conditions. The proof uses energy inequalities and the density of the range of the operator generated.

\section{Introduction}

In the rectangle $\Omega=(0,1) \times(0, T)$, we consider the equation

$$
f(x, t)=\frac{\partial^{3} u}{\partial t^{3}}+\frac{\partial}{\partial x}\left(a(x, t) \frac{\partial u}{\partial x}\right)
$$

with the initial conditions

$$
u(x, 0)=0, \quad \frac{\partial u}{\partial t}(x, 0)=0, \quad x \in(0,1),
$$

the final condition

$$
\frac{\partial^{2} u}{\partial t^{2}}(x, T)=0, \quad x \in(0,1)
$$

the Dirichlet condition

$$
u(0, t)=0 \quad \forall t \in(0, T),
$$

and the integral condition

$$
\int_{l}^{1} u(x, t) d x=0, \quad 0 \leq l<1, t \in(0, T) .
$$


In addition, we assume that the function $a(x, t)$ and its derivatives satisfy the conditions

$$
\begin{gathered}
0<a_{0}<a(x, t)<a_{1} \quad \forall x, t \in \Omega, \\
\left|\frac{\partial a}{\partial x}\right| \leq b \quad \forall x, t \in \Omega, \\
c_{k}^{\prime}<\frac{\partial^{k} u}{\partial t^{k}}(x, t)<c_{k} \quad \forall x, t \in \Omega, k=\overline{1,3} \text {, with } c_{1}^{\prime}>0 .
\end{gathered}
$$

Over the last few years, many physical phenomena were formulated into nonlocal mathematical models with integral boundary conditions $[1,9,10,11]$. The reader should refer to $[13,14]$ and the references therein. The importance of these kinds of problems has also been pointed out by Samarskii [22]. This type of boundary value problems has been investigated in $[2,3,4,6,7,8,12,18,19,20,23,25]$ for parabolic equations, in $[21,24]$ for hyperbolic equations, and in $[15,16,17]$ for mixed-type equations. The basic tool in $[5,15,16,17,20,25]$ is the energy inequality method which, of course, requires appropriate multipliers and functional spaces. In this paper, we extend this method to the study of a linear third-order partial differential equation.

\section{Preliminairies}

In this paper, we prove the existence and uniqueness of a strong solution of the problem (1.1)-(1.5). For this, we consider the solution of problem (1.1)-(1.5) as a solution of the operator equation

$$
L u=\mathscr{F},
$$

where the operator $L$ has domain of definition $D(L)$ consisting of functions $u \in L^{2}(\Omega)$ such that $\left(\partial^{k+1} u / \partial t^{k} \partial x\right)(x, t) \in L^{2}(\Omega), k=\overline{1,3}$ and satisfing the conditions (1.4)-(1.5).

The operator $L$ is considered from $E$ to $F$, where $E$ is the Banach space consisting of function $u \in L^{2}(\Omega)$, with the finite norm

$$
\begin{aligned}
\|u\|_{E}^{2}= & \int_{\Omega} \Theta(x)\left[\left|\frac{\partial^{3} u}{\partial t^{3}}\right|^{2}+\left|\frac{\partial^{2} u}{\partial x^{2}}\right|^{2}\right] d x d t \\
& +\int_{\Omega} \Theta(x)\left[\left|\frac{\partial u}{\partial x}\right|^{2}+\left|\frac{\partial^{2} u}{\partial t \partial x}\right|^{2}\right] d x d t \\
& +\int_{\Omega} \Phi(x)\left[\left|\frac{\partial u}{\partial t}\right|^{2}+|u|^{2}\right] d x d t .
\end{aligned}
$$

$F$ is the Hilbert space of functions $\mathscr{F}=(f, 0,0,0), f \in L^{2}(\Omega)$, with the finite norm

$$
\|\mathscr{F}\|_{F}^{2}=\int_{\Omega} \Theta(x)|f(x, t)|^{2} d x d t,
$$


where

$$
\begin{aligned}
& \Theta(x)= \begin{cases}(1-l)^{2}, & 0<x \leq l, \\
(1-x)^{2}, & l \leq x<1,\end{cases} \\
& \Phi(x)= \begin{cases}0, & 0<x<l, \\
1, & l \leq x<1 .\end{cases}
\end{aligned}
$$

\section{An energy inequality and its application}

Theorem 3.1. For any function $u \in D(L)$, the a priori estimate

$$
\|u\|_{E} \leq k\|L u\|_{F} \quad \text { for } u \in D(L)
$$

where $k^{2}=40 \exp (c T) / k_{1}$ with $k_{1}=\inf \left\{1 / 4,\left(c_{3}^{\prime}-3 c c_{1}^{\prime}+3 c^{2} c_{1}^{\prime}-c^{3} a_{1}-b^{2}\right) / 2, a_{0}^{2} / 2\right.$, (3/ 2) $\left.\left(c a_{0}-c_{1}\right)\right\}$. The constant $c$ satisfies

$$
\begin{gathered}
\sup _{(x, t) \in \Omega}\left(\frac{1}{a} \frac{\partial a}{\partial t}\right)<c<\inf _{(x, t) \in \Omega}\left(\frac{1}{a} \frac{\partial a}{\partial t}+1\right), \\
c_{3}^{\prime}-3 c c_{1}^{\prime}+3 c^{2} c_{1}^{\prime}-c^{3} a_{1}-b^{2}>0 \\
c_{2}^{\prime}-2 c c_{1}^{\prime}+c^{2} a_{1}^{2}+c a_{0}-c_{1}>0
\end{gathered}
$$

Proof. Let

$$
M u= \begin{cases}(1-l)^{2} \frac{\partial^{3} u}{\partial t^{3}}, & 0<x<l \\ (1-x)^{2} \frac{\partial^{3} u}{\partial t^{3}}+2(1-x) J_{x} \frac{\partial^{3} u}{\partial t^{3}}, & l<x<1\end{cases}
$$

where $J_{x} u=\int_{l}^{x} u(x, t) d x$.

We consider the quadratic form obtained by multiplying (1.1) by $\exp (-c t) \overline{M u}$, with the constant $c$ satisfying (3.2), integrating over $\Omega=(0,1) \times(0, T)$, and taking the real part:

$$
\Phi(u, u)=\operatorname{Re} \int_{\Omega} \exp (-c t) f(x, t) \overline{M u} d x d t
$$


By substituting the expression of $M u$ in (3.4), integrating with respect to $x$, and using the Dirichlet and integral conditions, we obtain

$$
\begin{aligned}
\operatorname{Re} \int_{\Omega} & \exp (-c t) f(x, t) \overline{M u} d x d t \\
= & \int_{0}^{T} \int_{0}^{1} \Theta(x) \exp (-c t)\left|\frac{\partial^{3} u}{\partial t^{3}}\right|^{2} d x d t \\
& -\frac{3}{2} \int_{0}^{T} \int_{0}^{1} \Theta(x) \exp (-c t)\left[\frac{\partial a}{\partial t}-c a\right]\left|\frac{\partial^{2} u}{\partial x \partial t}\right|^{2} d x d t \\
& +\int_{0}^{T} \int_{0}^{1} \frac{\Theta(x)}{2} \exp (-c t)\left[\frac{\partial^{3} a}{\partial t^{3}}-3 c \frac{\partial^{2} a}{\partial t^{2}}+3 c \frac{\partial a}{\partial t}-c^{3} a\right]\left|\frac{\partial u}{\partial x}\right|^{2} d x d t \\
& +\int_{0}^{T} \int_{l}^{1} \exp (-c t)\left|J_{x} \frac{\partial^{3} u}{\partial t^{3}}\right|^{2} d x d t \\
& -2 \operatorname{Re} \int_{0}^{T} \int_{l}^{1} \exp (-c t) a(x, t) u \frac{\partial^{3} u}{\partial t^{3}} d x d t \\
& +\left.\int_{0}^{1} \Theta(x) \exp (-c t) a(x, t)\left|\frac{\partial^{2} u}{\partial x \partial t}\right|^{2} d x\right|_{t=T} \\
& -\left.\int_{0}^{1} \Theta(x) \exp (-c t)\left(\frac{\partial a}{\partial t}-c a\right) \frac{\partial u}{\partial x} \frac{\partial^{2} u}{\partial x \partial t} d x\right|_{t=T} \\
& -\left.\int_{0}^{1} \frac{\Theta(x)}{2} \exp (-c t)\left[\frac{\partial^{2} a}{\partial t^{2}}-2 c \frac{\partial a}{\partial t}+c^{2} a\right]\left|\frac{\partial u}{\partial x}\right|^{2} d x\right|_{t=T} \\
& -2 \operatorname{Re} \int_{0}^{T} \int_{l}^{1} \exp (-c t) \frac{\partial a}{\partial x} u J_{x} \frac{\partial^{3} u}{\partial t^{3}} d x d t .
\end{aligned}
$$

Integrating by parts $-2 \operatorname{Re} \int_{0}^{T} \int_{l}^{1} \exp (-c t) a(x, t) u\left(\overline{\partial^{3} u} / \partial t^{3}\right) d x d t$ with respect to $t$, and using the initial conditions, the final conditions, and the elementary inequalities, we obtain

$$
\begin{aligned}
\int_{0}^{T} \int_{0}^{1} \frac{\Theta}{2}(x) & \exp (-c t)\left|\frac{\partial^{3} u}{\partial t^{3}}\right|^{2} d x d t \\
& -\frac{3}{2} \int_{0}^{T} \int_{0}^{1} \Theta(x) \exp (-c t)\left[\frac{\partial a}{\partial t}-c a\right]\left|\frac{\partial^{2} u}{\partial x \partial t}\right|^{2} d x d t \\
& +\int_{0}^{T} \int_{0}^{1} \frac{\Theta(x)}{2} \exp (-c t)\left[\frac{\partial^{3} a}{\partial t^{3}}-3 c \frac{\partial^{2} a}{\partial t^{2}}+3 c \frac{\partial a}{\partial t}-c^{3} a\right]\left|\frac{\partial u}{\partial x}\right|^{2} d x d t \\
& +\int_{0}^{T} \int_{l}^{1} \exp (-c t)\left|J_{x} \frac{\partial^{3} u}{\partial t^{3}}\right|^{2} d x d t \\
& +\int_{0}^{T} \int_{l}^{1} \exp (-c t)\left[\frac{\partial^{3} a}{\partial t^{3}}-3 c \frac{\partial^{2} a}{\partial t^{2}}+3 c \frac{\partial a}{\partial t}-c^{3} a\right]|u|^{2} d x d t \\
& -\frac{3}{2} \int_{0}^{T} \int_{l}^{1} \exp (-c t)\left[\frac{\partial a}{\partial t}-c a\right]\left|\frac{\partial u}{\partial t}\right|^{2} d x d t \\
& +\left.\int_{0}^{1} \frac{\Theta(x)}{2} \exp (-c t)\left[a-\left|\frac{\partial a}{\partial t}-c a\right|\right]\left|\frac{\partial^{2} u}{\partial x \partial t}\right|^{2} d x\right|_{t=T}
\end{aligned}
$$




$$
\begin{aligned}
& -\left.\int_{0}^{1} \frac{\Theta(x)}{2} \exp (-c t)\left[\frac{\partial^{2} a}{\partial t^{2}}-2 c \frac{\partial a}{\partial t}+c^{2} a+\left|\frac{\partial a}{\partial t}-c a\right|\right]\left|\frac{\partial u}{\partial x}\right|^{2} d x\right|_{t=T} \\
& +\left.\int_{0}^{1} \Phi(x) \exp (-c t)\left[a-\left|\frac{\partial a}{\partial t}-c a\right|\right]\left|\frac{\partial u}{\partial t}\right|^{2} d x\right|_{t=T} \\
& -\left.\int_{0}^{1} \Phi(x) \exp (-c t)\left[\frac{\partial^{2} a}{\partial t^{2}}-2 c \frac{\partial a}{\partial t}+c^{2} a+\left|\frac{\partial a}{\partial t}-c a\right|\right]|u|^{2} d x\right|_{t=T} \\
& \leq 17 \int_{0}^{T} \int_{l}^{1} \Theta(x) \exp (-c t)|f|^{2} d x d t .
\end{aligned}
$$

From (1.1), we get

$$
\begin{aligned}
\int_{\Omega} \Theta(x) a^{2}\left|\frac{\partial^{2} u}{\partial x^{2}}\right|^{2} d x d t & \\
\leq & 2 \int_{\Omega} \Theta(x)\left|\frac{\partial^{3} u}{\partial t^{3}}\right|^{2} d x d t+2 \int_{\Omega} \Theta(x)\left(\frac{\partial a}{\partial x}\right)^{2}\left|\frac{\partial u}{\partial x}\right|^{2} d x d t \\
& +4 \int_{\Omega} \Theta(x)|f|^{2} d x d t
\end{aligned}
$$

Combining this last inequality with (3.6) and using the conditions (3.2) yield

$$
\begin{aligned}
\int_{\Omega} \Theta(x) & {\left[\left|\frac{\partial^{3} u}{\partial t^{3}}\right|^{2}+\left|\frac{\partial^{2} u}{\partial x^{2}}\right|^{2}\right] d x d t } \\
& +\int_{\Omega} \Theta(x)\left[\left|\frac{\partial u}{\partial x}\right|^{2}+\left|\frac{\partial^{2} u}{\partial t \partial x}\right|^{2}\right] d x d t+\int_{\Omega} \Phi(x)\left[\left|\frac{\partial u}{\partial t}\right|^{2}+|u|^{2}\right] d x d t \\
\leq & k \int_{\Omega} \Theta(x)|f(x, t)|^{2} d x d t
\end{aligned}
$$

which is the desired inequality.

It can be proved in a standard way that the operator $L: E \rightarrow F$ is closable. Let $\bar{L}$ be the closure of this operator, with the domain of definition $D(\bar{L})$.

Definition 3.2. A solution of the operator equation $\bar{L} u=\mathscr{F}$ is called a strong solution of problem (1.1)-(1.5).

The a priori estimate (3.1) can be extended to strong solutions, that is, we have the estimate

$$
\|u\|_{E} \leq c\|\bar{L} u\|_{F} \quad \forall u \in D(\bar{L})
$$

This last inequality implies the following corollaries.

Corollary 3.3. A strong solution of (1.1)-(1.5) is unique and depends continuously on $\mathscr{F}$. Corollary 3.4. The range $R(\bar{L})$ of $\bar{L}$ is closed in $F$ and $\overline{R(L)}=R(\bar{L})$. 
Corollary 3.4 shows that to prove that problem (1.1)-(1.5) has a strong solution for arbitrary $\mathscr{F}$, it suffices to prove that set $R(L)$ is dense in $F$.

\section{Solvability of problem (1.1)-(1.5)}

To prove the solvability of problem (1.1)-(1.5) it is sufficient to show that $R(L)$ is dense in $F$. The proof is based on the following lemma.

Lemma 4.1. Suppose that the function $a(x, t)$ and its derivatives are bounded. Let $u \in D_{0}(L)$ $=\left\{u \in D(L), u(x, 0)=0,(\partial u / \partial t)(x, 0)=0,\left(\partial^{2} u / \partial t^{2}\right)(x, T)=0\right\}$. If for $u \in D_{0}(L)$ and some functions $w(x, t) \in L^{2}(\Omega)$,

$$
\int_{\Omega} h(x) f \bar{w} d x d t=0
$$

where

$$
h(x)= \begin{cases}1-l, & 0<x<l \\ 1-x, & l<x<1,\end{cases}
$$

holds, for arbitrary $u \in D_{0}(L)$, and then $w=0$.

Proof. The equality (4.1) can be written as follows:

$$
\int_{\Omega} h(x) \frac{\partial^{3} u}{\partial t^{3}} \bar{w} d x d t=\int_{\Omega} A(t) u \bar{v} d x d t
$$

for a given $w(x, t)$, where

$$
\begin{aligned}
& v= \begin{cases}(1-l) w, & 0<x<l, \\
w-\int_{l}^{x} \frac{w}{1-\zeta} d \zeta, & l<x<1,\end{cases} \\
& A(t) u=\frac{\partial}{\partial x}\left(h(x) a(x, t) \frac{\partial u}{\partial x}\right), \\
& N v= \begin{cases}(1-l) v, & 0<x<l, \\
(1-x) v+J_{x} v, & l<x<1 .\end{cases}
\end{aligned}
$$

For $v=w-\int_{l}^{x}(w /(1-\zeta)) d \zeta, l<x<1$ we deduce $\int_{l}^{x} v(\zeta, t) d \zeta=(1-x) \int_{l}^{x}(w /(1-\zeta)) d \zeta$, then $\int_{l}^{1} v(\zeta, t) d \zeta=0$.

Following [25], we introduce the smoothing operators with respect to $t,\left(J_{\epsilon}^{-1}\right)=(I-$ $\left.\epsilon\left(\partial^{3} / \partial t^{3}\right)\right)^{-1}$, and $\left(J_{\epsilon}^{-1}\right)^{*}=\left(I+\epsilon\left(\partial^{3} / \partial t^{3}\right)\right)^{-1}$ which provide the solution of the respective problems:

$$
\begin{array}{llll}
u_{\epsilon}-\epsilon \frac{\partial^{3} u_{\epsilon}}{\partial t^{3}}=u, & u_{\epsilon}(x, 0)=0, & \frac{\partial u_{\epsilon}}{\partial t}(x, 0)=0, & \frac{\partial^{2} u_{\epsilon}}{\partial t^{2}}(x, T)=0, \\
v_{\epsilon}^{*}+\epsilon \frac{\partial^{3} v_{\epsilon}^{*}}{\partial t^{3}}=v, & v_{\epsilon}^{*}(x, 0)=0, & \frac{\partial v_{\epsilon}^{*}}{\partial t}(x, T)=0, & \frac{\partial^{2} v_{\epsilon}^{*}}{\partial t^{2}}(x, T)=0 .
\end{array}
$$


And also, we have the following properties: for any $u \in L^{2}(0, T)$, the function $J_{\epsilon}^{-1} u \in$ $W_{2}^{3}(0, T),\left(J_{\epsilon}^{-1}\right)^{*} u \in W_{2}^{3}(0, T)$. If $u \in D(L), J_{\epsilon}^{-1} u \in D(L)$.

$$
\lim _{\epsilon \rightarrow 0}\left\|J_{\epsilon}^{-1} u-u\right\|_{L^{2}(0, T)}=0, \quad \lim _{\epsilon \rightarrow 0}\left\|\left(J_{\epsilon}^{-1}\right)^{*} u-u\right\|_{L^{2}(0, T)}=0
$$

Substituting the function $u$ in (4.3) by the smoothing function $u_{\epsilon}$ and using the relation $A(t) u_{\epsilon}=J_{\epsilon}^{-1} A(t) u+\epsilon J_{\epsilon}^{-1} B_{\epsilon}(t) u$, where $B_{\epsilon}(t)=(3 \partial / \partial t)\left((\partial A(t) / \partial t)\left(\partial u_{\epsilon} / \partial t\right)\right)+\left(\partial^{3} A(t) /\right.$ $\left.\partial t^{3}\right) u_{\epsilon}$, we obtain

$$
\int_{\Omega} u \overline{\frac{\partial^{3} v_{\epsilon}^{*}}{\partial t^{3}}} d x d t=\int_{\Omega} A(t) \overline{u v_{\epsilon}^{*}} d x d t-\epsilon \int_{\Omega} B_{\epsilon}(t) \overline{u v_{\epsilon}^{*}} d x d t
$$

The operator $A(t)$ has a continuous inverse in $L^{2}(0,1)$ defined by

$$
A^{-1}(t) g= \begin{cases}-\frac{1}{1-l} \int_{0}^{x} \frac{d \zeta}{a(\zeta, t)} \int_{0}^{\zeta} g(\eta) d \eta+\frac{C_{1}(t)}{1-l} \int_{0}^{x} \frac{d \zeta}{a(\zeta, t)}, & 0<x<l \\ \int_{l}^{x} \frac{-d \zeta}{(1-\zeta) a(\zeta, t)} \int_{l}^{\zeta} g(\eta) d \eta+C_{2}(t) \int_{l}^{x} \frac{d \zeta}{(1-\zeta) a(\zeta, t)}+u(l), & l<x<1\end{cases}
$$

where

$$
\begin{aligned}
C_{1}(t) & =\frac{(1-l) u(l)+\int_{0}^{l}(d \zeta / a(\zeta, t)) \int_{0}^{\zeta} g(\eta) d \eta}{\int_{0}^{l}(d \zeta / a(\zeta, t))} \\
C_{2}(t) & =\frac{-(1-l) u(l)+\int_{l}^{1}(d \zeta / a(\zeta, t)) \int_{l}^{\zeta} g(\eta) d \eta}{\int_{l}^{1}(d \zeta / a(\zeta, t))}
\end{aligned}
$$

Then we have $\int_{l}^{1} A^{-1}(t) u=0$, hence, the function $J_{\epsilon}^{-1} u=u_{\varepsilon}$ can be represented in the form

$$
u_{\varepsilon}=J_{\epsilon}^{-1} A^{-1}(t) A(t) u
$$

The adjoint of $B_{\epsilon}(t)$ has the form

$$
\begin{aligned}
B_{\epsilon}^{*}(t) v= & \frac{1}{a}\left(J_{\epsilon}^{-1}\right) * \frac{\partial^{3} a}{\partial t^{3}} v+\frac{3}{a}\left(J_{\epsilon}^{-1}\right) * \frac{\partial}{\partial t}\left(\frac{\partial a}{\partial t} \frac{\partial v}{\partial t}\right)-G_{\epsilon}(v)(x) \\
& +\frac{\int_{0}^{x}(d \zeta / a(\zeta, t))}{\int_{0}^{1}(d \zeta / a(\zeta, t))} G_{\epsilon}(v)(1),
\end{aligned}
$$

where

$$
\begin{aligned}
G_{\epsilon}(v)(x)=\int_{0}^{x}[ & \frac{3}{a}\left(J_{\epsilon}^{-1}\right)^{*} \frac{\partial}{\partial t}\left(\frac{\partial^{2} a}{\partial t \partial \zeta} \frac{\partial v}{\partial t}\right)-\frac{3}{a^{2}} \frac{\partial a}{\partial \zeta}\left(J_{\epsilon}^{-1}\right)^{*} \frac{\partial}{\partial t}\left(\frac{\partial a}{\partial t} \frac{\partial v}{\partial t}\right) \\
& \left.+\frac{1}{a}\left(J_{\epsilon}^{-1}\right)^{*} \frac{\partial}{\partial t}\left(\frac{\partial^{4} a}{\partial t^{3} \partial \zeta} v\right)-\frac{1}{a^{2}} \frac{\partial a}{\partial \zeta}\left(J_{\epsilon}^{-1}\right)^{*}\left(\frac{\partial^{3} a}{\partial t^{3}} v\right)\right] d \zeta
\end{aligned}
$$


Consequently, equality (4.7) becomes

$$
\int_{\Omega} u N \frac{\overline{\partial^{3} v_{\epsilon}^{*}}}{\partial t^{3}} d x d t=\int_{\Omega} A(t) u \overline{h_{\epsilon}} d x d t
$$

where $h_{\epsilon}=v_{\epsilon}^{*}-\epsilon B_{\epsilon}^{*}(t) v_{\epsilon}^{*}$.

The left-hand side of (4.13) is a continuous linear functional of $u$, hence the function $h_{\epsilon}$ has the derivatives $\partial h_{\epsilon} / \partial x,(1-x)\left(\partial h_{\epsilon} / \partial x\right) \in L^{2}(\Omega)$, and the condition $h_{\epsilon}(0, t)=0$ is satisfied.

From the equality

$$
(1-x) \frac{\partial h_{\epsilon}}{\partial x}=\left[I-\epsilon \frac{1}{a}\left(J_{\epsilon}^{-1}\right)^{*}\left(\frac{\partial^{3} a}{\partial t^{3}}\right)\right](1-x) \frac{\partial v_{\epsilon}^{*}}{\partial x}-3 \epsilon \frac{1}{a}\left(J_{\epsilon}^{-1}\right)^{*} \frac{\partial}{\partial t}\left(\frac{\partial a}{\partial t} \frac{\partial}{\partial t}(1-x) \frac{\partial v_{\epsilon}^{*}}{\partial x}\right)
$$

and since the operator $\left(J_{\epsilon}^{-1}\right)^{*}$ is bounded in $L^{2}(\Omega)$, for sufficiently small $\epsilon$, we have $\left\|\epsilon(1 / a)\left(J_{\epsilon}^{-1}\right)^{*}\left(\partial^{3} a / \partial t^{3}\right)\right\|<1$. Hence, the operator $I-\epsilon(1 / a)\left(J_{\epsilon}^{-1}\right)^{*}\left(\partial^{3} a / \partial t^{3}\right)$ has a bounded inverse in $L^{2}(\Omega)$. We conclude that $(1-x)\left(\partial v_{\epsilon}^{*} / \partial x\right) \in L^{2}(\Omega)$. Similarly, we conclude that $(\partial / \partial x)\left((1-x)\left(\partial v_{\epsilon}^{*} / \partial x\right)\right)$ exists and belongs to $L^{2}(\Omega)$, and the condition $v_{\epsilon}^{*}(0, t)=0$ is satisfied.

Putting $u=\int_{0}^{t} \int_{0}^{\zeta} \int_{\eta}^{T} \exp (c \tau) v_{\epsilon}^{*} d \tau d \eta d \zeta$ in (4.3), where the constant $c$ satisfies (3.2) and using the proprieties of smoothing operator, we obtain

$$
\int_{\Omega} \exp (c t) v_{\varepsilon}^{*} \overline{N v} d x d t=-\int_{\Omega} A(t) u \overline{v_{\varepsilon}^{*}} d x d t-\varepsilon \int_{\Omega} A(t) u \frac{\overline{\partial^{3} v_{\epsilon}^{*}}}{\partial t^{3}} d x d t
$$

and from

$$
\begin{aligned}
-\varepsilon \int_{\Omega} A(t) u \frac{\overline{\partial^{3} v_{\epsilon}^{*}}}{\partial t^{3}} d x d t \\
=3 \int_{\Omega} h(x) \exp (-c t) \frac{\partial^{2} a}{\partial t^{2}}\left|\frac{\partial^{3} u}{\partial t^{2} \partial x}\right|^{2} d x d t \\
\quad-3 \int_{\Omega} h(x) \exp (-c t)\left[\frac{\partial^{3} a}{\partial t^{3}}-c \frac{\partial^{2} a}{\partial t^{2}}\right] \frac{\partial^{3} u}{\partial t^{2} \partial x} \frac{\partial^{2} u}{\partial t \partial x} d x d t \\
+\left.3 \int_{0}^{1} \frac{h(x)}{2} \exp (-c t) \frac{\partial a}{\partial t}\left|\frac{\partial^{3} u}{\partial t^{2} \partial x}\right|^{2} d x\right|_{t=T} \\
+\left.3 \int_{0}^{1} \frac{h(x)}{2} \exp (-c t)\left[\frac{\partial^{2} a}{\partial t^{2}}-c \frac{\partial a}{\partial t}\right]\left|\frac{\partial^{2} u}{\partial t \partial x}\right|^{2} d x\right|_{t=T} \\
\quad-\int_{\Omega} h(x) \exp (-c t) a\left|\frac{\partial^{3} v_{\epsilon}^{*}}{\partial t^{3}}\right|^{2} d x d t \\
\quad-\int_{\Omega} h(x) \exp (-c t) \frac{\partial^{3} a}{\partial t^{3}} \frac{\partial u}{\partial x} \frac{\partial^{3} u}{\partial t^{2} \partial x} d x d t
\end{aligned}
$$


we have

$$
\begin{aligned}
&-\varepsilon \operatorname{Re} \int_{\Omega} A(t) u \frac{\partial^{3} v_{\epsilon}^{*}}{\partial t^{3}} d x d t \\
& \leq \varepsilon\left\{3 \int_{\Omega} h(x) \exp (-c t)\left[\frac{\partial^{2} a}{\partial t^{2}}+\frac{1}{2}\left|\frac{\partial^{3} a}{\partial t^{3}}-c \frac{\partial^{2} a}{\partial t^{2}}\right|\right]\left|\frac{\partial^{3} u}{\partial t^{2} \partial x}\right|^{2} d x d t\right. \\
&+\frac{3}{2} \int_{\Omega} h(x) \exp (-c t)\left[\frac{\partial^{2} a}{\partial t^{2}}-c \frac{\partial a}{\partial t}+\left|\frac{\partial^{3} a}{\partial t^{3}}-c \frac{\partial^{2} a}{\partial t^{2}}\right|\right]\left|\frac{\partial^{2} u}{\partial t \partial x}\right|^{2} d x d t \\
&-\int_{\Omega} h(x) \exp (-c t) a\left|\frac{\partial^{3} v_{\epsilon}^{*}}{\partial t^{3}}\right|^{2} d x d t \\
&+\frac{3}{2} \int_{\Omega} h(x) \exp (-c t)\left|\frac{\partial^{3} a}{\partial t^{3}}\right|\left|\frac{\partial u}{\partial x}\right|^{2} d x d t \\
&+\frac{1}{2} \int_{\Omega} h(x) \exp (-c t)\left|\frac{\partial^{3} a}{\partial t^{3}}\right|\left|\frac{\partial^{4} u}{\partial t^{3} \partial x}\right|^{2} d x d t \\
&\left.+\frac{1}{2} \int_{\Omega} h(x) \exp (-c t) \frac{\partial a}{\partial t}\left|\frac{\partial^{3} u}{\partial t^{2} \partial x}\right|^{2} d x d t\right\} .
\end{aligned}
$$

Integrating the first term on the right-hand side by parts in (4.15), we obtain

$$
\begin{aligned}
&-\varepsilon \operatorname{Re} \int_{\Omega} A(t) u \overline{v_{\varepsilon}^{*}} d x d t \\
&=\frac{3}{2} \int_{\Omega} h(x) \exp (-c t)\left[\frac{\partial a}{\partial t}-c a\right]\left|\frac{\partial^{2} u}{\partial t \partial x}\right|^{2} d x d t \\
&-\int_{\Omega} h(x) \exp (-c t)\left\{\frac{\partial^{3} a}{\partial t^{3}}-3 c \frac{\partial^{2} a}{\partial t^{2}}+3 c^{2} \frac{\partial a}{\partial t}-c^{3} a\right\}\left|\frac{\partial u}{\partial x}\right|^{2} d x d t \\
&-\left.\int_{0}^{1} \frac{1}{2} h(x) \exp (-c t) a\left|\frac{\partial^{2} u}{\partial t \partial x}\right|^{2} d x\right|_{t=T} \\
&+\left.\int_{0}^{1} \frac{1}{2} h(x) \exp (-c t)\left\{\frac{\partial^{2} a}{\partial t^{2}}-2 c \frac{\partial a}{\partial t}+c^{2} a\right\}\left|\frac{\partial u}{\partial x}\right|^{2} d x\right|_{t=T} \\
&-\left.\int_{0}^{1} h(x) \exp (-c t)\left\{\frac{\partial a}{\partial t}-c a\right\} \frac{\partial u}{\partial x} \frac{\partial^{2} u}{\partial t \partial x} d x\right|_{t=T} .
\end{aligned}
$$

This last equality gives

$$
\begin{aligned}
-\varepsilon \operatorname{Re} \int_{\Omega} A(t) u \overline{v_{\varepsilon}^{*}} d x d t \\
\leq-\left.\int_{0}^{1} h(x) \exp (-c t)\left|\frac{\partial a}{\partial t}+a-c a\right|\left|\frac{\partial^{2} u}{\partial x \partial t}\right|^{2} d x\right|_{t=T} \\
\quad+\left.\int_{0}^{1} \frac{1}{2} h(x) \exp (-c t)\left\{\frac{\partial^{2} a}{\partial t^{2}}-2 c \frac{\partial a}{\partial t}+c^{2} a+c a-\frac{\partial a}{\partial t}\right\}\left|\frac{\partial u}{\partial x}\right|^{2} d x\right|_{t=T}
\end{aligned}
$$

By using the conditions (3.2), inequalities (4.17) and (4.19), we obtain

$$
\operatorname{Re} \int_{\Omega} \exp (c t) v_{\varepsilon}^{*} \overline{N v} d x d t \leq 0 \quad \text { as } \epsilon \longrightarrow 0 .
$$


This implies $\operatorname{Re} \int_{\Omega} \exp (c t)\left(v_{\varepsilon}^{*}-v\right) \overline{N v} d x d t+\operatorname{Re} \int_{\Omega} \exp (c t) v \overline{N v} d x d t \leq 0$, that is,

$$
\begin{aligned}
& \int_{0}^{T} \int_{0}^{l} \exp (-c t)(1-l)|v|^{2} d x d t \\
& \quad+\int_{0}^{T} \int_{l}^{1} \int_{0}^{l} \exp (-c t)(1-x)|v|^{2} d x d t+\int_{0}^{T} \int_{l}^{1} \exp (-c t)\left|J_{x} v\right|^{2} d x d t \\
& \quad+\int_{0}^{T} \int_{0}^{l} \frac{1-l}{2 l} \exp (-c t)\left|J_{x} v\right|^{2} d x d t \leq 0 .
\end{aligned}
$$

Then $v=0$.

Finally from (4.4), we conclude $w=0$.

Theorem 4.2. The range $R(\bar{L})$ of $\bar{L}$ coincides with $F$.

Proof. Since $F$ is Hilbert space, then $R(\bar{L})=F$ if and only if the relation

$$
\int_{\Omega} \Theta(x) f \bar{g} d x d t=0
$$

holds.

Arbitrary $u \in D_{0}(L)$ and $\mathscr{F}=(f, 0,0,0) \in F$ implies $f=0$. Taking in (4.22), $u \in D_{0}(L)$, and using Lemma 4.1, we obtain

$$
w= \begin{cases}(1-l) g, & 0<x<l \\ (1-x) g, & l<x<1\end{cases}
$$

then $g=0$.

\section{References}

[1] W. Allegretto, Y. Lin, and A. Zhou, A box scheme for coupled systems resulting from microsensor thermistor problems, Dynam. Contin. Discrete Impuls. Systems 5 (1999), no. 1-4, 209-223.

[2] G. W. Batten, Jr., Second-order correct boundary conditions for the numerical solution of the mixed boundary problem for parabolic equations, Math. Comp. 17 (1963), 405-413.

[3] S. A. Beilin, Existence of solutions for one-dimensional wave equations with nonlocal conditions, Electron. J. Differential Equations 2001 (2001), no. 76, 1-8.

[4] N.-E. Benouar and N. I. Yurchuk, Mixed problem with an integral condition for parabolic equations with the Bessel operator, Differ. Equ. 27 (1991), no. 12, 1482-1487.

[5] A. Bouziani and N.-E. Benouar, Mixed problem with integral conditions for a third order parabolic equation, Kobe J. Math. 15 (1998), no. 1, 47-58.

[6] B. Cahlon, D. M. Kulkarni, and P. Shi, Stepwise stability for the heat equation with a nonlocal constraint, SIAM J. Numer. Anal. 32 (1995), no. 2, 571-593.

[7] J. R. Cannon, The solution of the heat equation subject to the specification of energy, Quart. Appl. Math. 21 (1963), 155-160.

[8] The One-Dimensional Heat Equation, Encyclopedia of Mathematics and its Applications, vol. 23, Addison-Wesley Publishing, Massachusetts, 1984.

[9] J. R. Cannon, Y. Lin, and S. Wang, An implicit finite difference scheme for the diffusion equation subject to mass specification, Internat. J. Engrg. Sci. 28 (1990), no. 7, 573-578.

[10] J. R. Cannon and A. L. Matheson, A numerical procedure for diffusion subject to the specification of mass, Internat. J. Engrg. Sci. 31 (1993), no. 3, 347-355. 
[11] V. Capasso and K. Kunisch, A reaction-diffusion system arising in modelling man-environment diseases, Quart. Appl. Math. 46 (1988), no. 3, 431-450.

[12] Y. S. Choi and K.-Y. Chan, A parabolic equation with nonlocal boundary conditions arising from electrochemistry, Nonlinear Anal. 18 (1992), no. 4, 317-331.

[13] J. H. Cushman and T. R. Ginn, Nonlocal dispersion in porous media with continuously evolving scales of heterogeneity, J. Transport in Porous Media 13 (1993), no. 1, 123-138.

[14] J. H. Cushman, H. Xu, and F. Deng, Nonlocal reactive transport with physical and chemical heterogeneity: localization error, Water Resources Res. 31 (1995), no. 9, 2219-2237.

[15] M. Denche and A. L. Marhoune, High-order mixed-type differential equations with weighted integral boundary conditions, Electron. J. Differential Equations 2000 (2000), no. 60, 1-10.

[16] - Mixed problem with nonlocal boundary conditions for a third-order partial differential equation of mixed type, Int. J. Math. Math. Sci. 26 (2001), no. 7, 417-426.

[17] - Mixed problem with integral boundary condition for a high order mixed type partial differential equation, J. Appl. Math. Stochastic Anal. 16 (2003), no. 1, 69-79.

[18] N. I. Ionkin, The solution of a certain boundary value problem of the theory of heat conduction with a nonclassical boundary condition, Differ. Uravn. 13 (1977), no. 2, 294-304 (Russian).

[19] L. I. Kamynin, A boundary value problem in the theory of heat conduction with a nonclassical boundary condition, Comput. Math. Math. Phys. 4 (1964), no. 6, 33-59.

[20] A. V. Kartynnik, Three-point boundary-value problem with an integral space-variable condition for a second-order parabolic equation, Differ. Equ. 26 (1990), no. 9, 1160-1166.

[21] L. S. Pulkina, A non-local problem with integral conditions for hyperbolic equations, Electron. J. Differential Equations 1999 (1999), no. 45, 1-6.

[22] A. A. Samarski, Some problems in the modern theory of differential equations, Differ. Uravn. 16 (1980), 1925-1935 (Russian).

[23] P. Shi, Weak solution to an evolution problem with a nonlocal constraint, SIAM J. Math. Anal. 24 (1993), no. 1, 46-58.

[24] V. F. Volkodavov and V. E. Zhukov, Two problems for the string vibration equation with integral conditions and special matching conditions on the characteristic, Differ. Equ. 34 (1998), no. 4, 501-505.

[25] N. I. Yurchuk, Mixed problem with an integral condition for certain parabolic equations, Differ. Equ. 22 (1986), 1457-1463.

C. Latrous: Laboratoire Equations Differentielles, Département de Mathematiques, Université Mentouri Constantine, 25000 Constantine, Algeria

E-mail address: clatrous@wissal.dz

A. Memou: Laboratoire Equations Differentielles, Département de Mathematiques, Université Mentouri Constantine, 25000 Constantine, Algeria

E-mail address: memoua@wissal.dz 


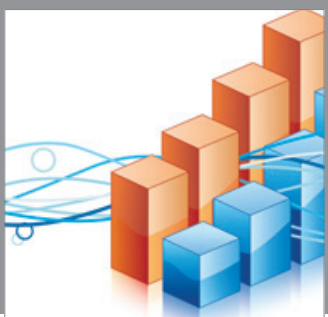

Advances in

Operations Research

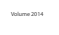

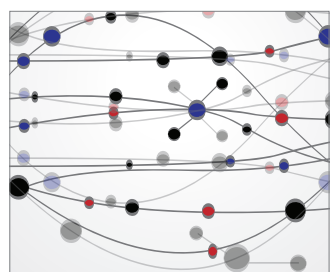

\section{The Scientific} World Journal
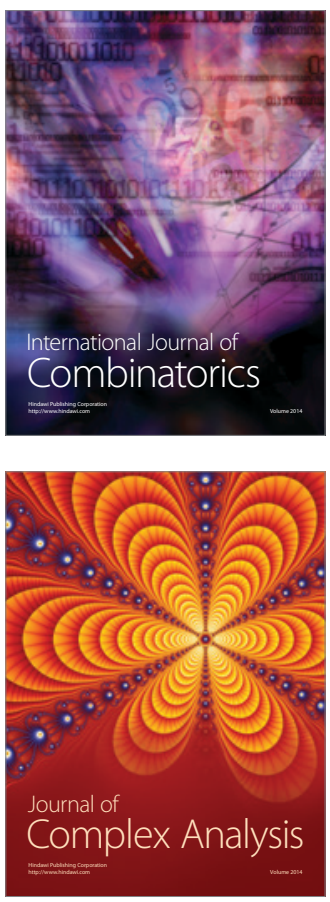

International Journal of

Mathematics and

Mathematical

Sciences
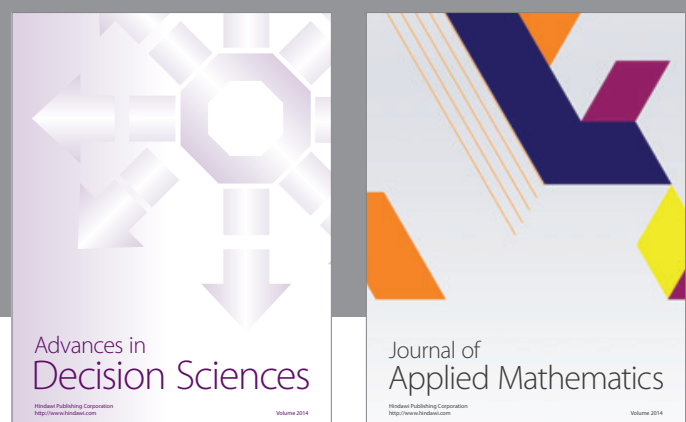

Journal of

Applied Mathematics
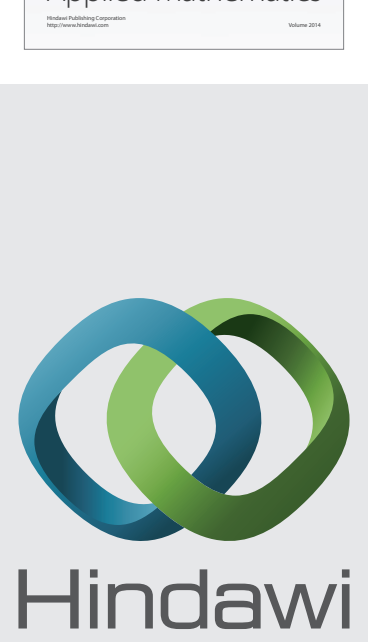

Submit your manuscripts at http://www.hindawi.com
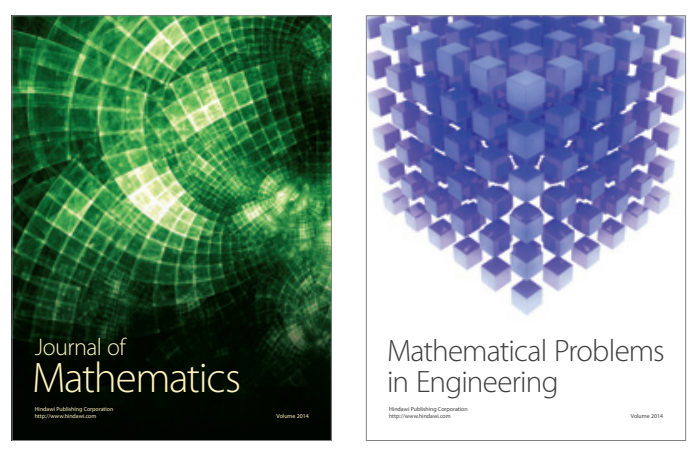

Mathematical Problems in Engineering
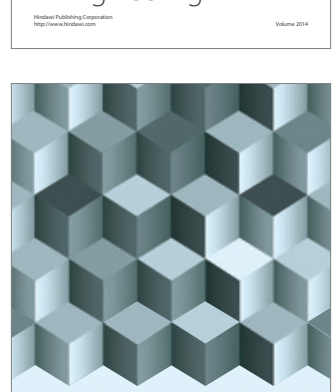

Journal of

Function Spaces
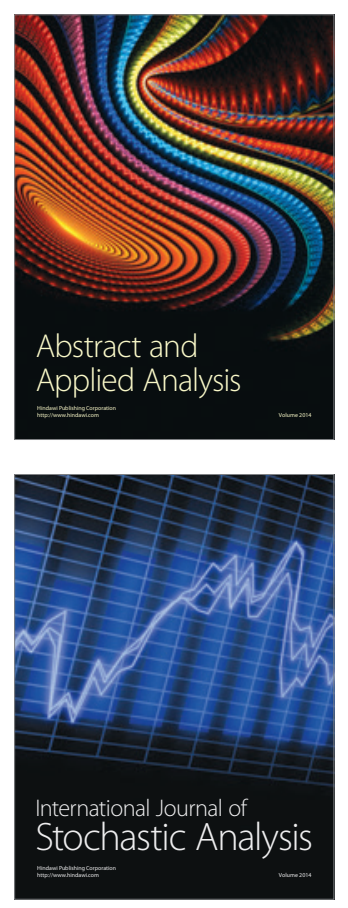

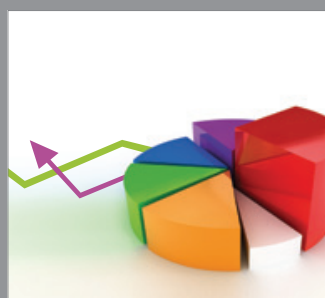

ournal of

Probability and Statistics

Promensencen
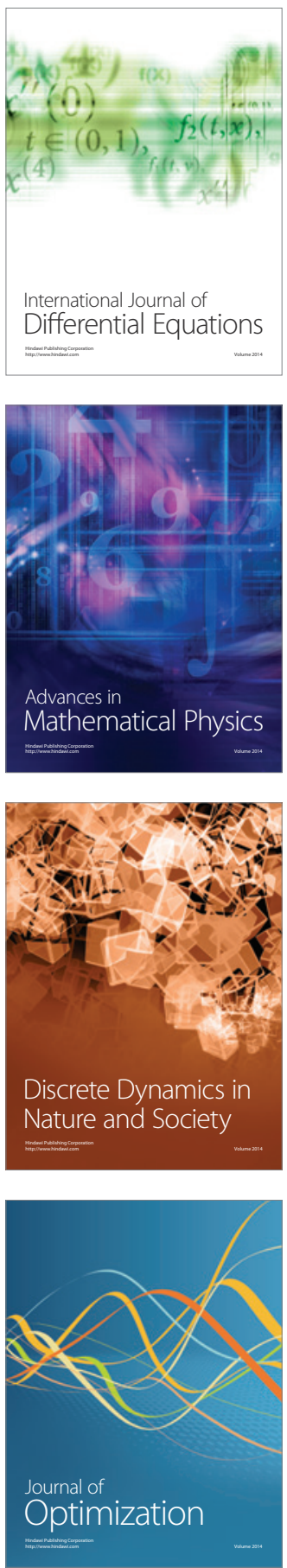